\title{
Resampling and Subsampling for Financial Time Series
}

\author{
Efstathios Paparoditis ${ }^{1}$ and Dimitris N. Politis ${ }^{2}$ \\ 1 University of Cyprus (stathisp@ucy.ac.cy) \\ ${ }^{2}$ University of California, San Diego (politis@math.ucsd.edu)
}

\section{Introduction}

Consider a discrete time process describing the behavior of log-returns

$$
R_{t}=\log \left(1+\frac{P_{t}-P_{t-1}}{P_{t-1}}\right), \quad t=1,2, \ldots
$$

where $\left\{P_{t}, t=0,1,2, \ldots\right\}$ is the price of a financial asset observed at time $t$, $t$ can be measured in seconds, minutes, hours, days, etc. Standard examples for $P_{t}$ are prices of company-shares quoted at major stock exchanges, interest rates and foreign exchange rates among different currencies. A Taylor series argument shows that $R_{t}$ is close to the relative returns $\left(P_{t}-P_{t-1}\right) / P_{t-1}$ which are free of unit and describe the relative change over time of the price process. Statistical inference for such financial time series has received considerable interest in the last decades leading to a vast and growing literature.

A general class of models proposed to describe the behavior of log returns is given by the following multiplicative model

$$
R_{t}=\mu_{t}+\sigma_{t} \cdot \varepsilon_{t}
$$

where $\mu_{t}$ and $\sigma_{t}$ are random variables measurable with respect to the $\sigma$-field $\mathcal{F}_{t-1}=\sigma\left(R_{t-j}, j=1,2, \ldots\right)$ and $\left\{\varepsilon_{t}\right\}$ denotes a sequence of i.i.d. random variables independent of $\left\{R_{t-j}, j \geq 1\right\}$ with zero mean and unit variance. It is also assumed that $\varepsilon_{t}$ is independent of the conditional (on the past logreturns $\left.R_{t-j}, j=1,2, \ldots\right)$ mean $\mu_{t}$ and of the conditional (non-negative) volatility function $\sigma_{t}^{2}$. By model (1) the direction of the price change at time $t$ is determined by the sign of $\varepsilon_{t}$, while the order of magnitude of this change by the volatility process $\sigma_{t}^{2}$ which is independent of $\varepsilon_{t}$. In what follows we assume for simplicity that $\mu_{t} \equiv 0$ and concentrate on bootstrap-based statistical inference for the conditional variance $\sigma_{t}^{2}$ of the log-returns.

Several of the statistical models proposed in the literature specify the volatility function $\sigma_{t}^{2}$ as a function of the observable past values $R_{t-j}$ and 
$\sigma_{t-j}^{2}, j=1,2, \ldots$ For instance, $\left\{R_{t}\right\}$ follows a general nonnegative $\operatorname{ARCH}(\infty)$ equation if for some known nonnegative function $w(\cdot)$,

$$
w\left(R_{t}\right)=\rho_{t} \xi_{t}
$$

where

$$
\rho_{t}=a+\sum_{j=1}^{\infty} \beta_{j} w\left(R_{t-1}\right),
$$

$\left\{\xi_{t}\right\}$ is a sequence of i.i.d. nonnegative random variables, $a \geq 0$ and $\beta_{j} \geq 0$, $j=1,2, \ldots$; see Robinson (1991), Giraitis et al. (2000) and Kazakevičius and Leipus (2002). $E \xi_{1}^{2}<\infty$ and $\sum_{j=1}^{\infty} \beta_{j}^{2}<1$ imply weak stationarity of $\rho_{t}$. The class (2)-(3) is rich enough and includes as special cases the classical $\operatorname{ARCH}(p)$ process (Engle (1982)) as well as the $\operatorname{ARCH}(\infty)$ process obtained for $w(x)=x^{2}$,

$$
R_{t}^{2}=\left(a+\sum_{j=1}^{\infty} \beta_{j} R_{t-j}^{2}\right) \varepsilon_{t}^{2} .
$$

Under certain assumptions on the behavior of the $b_{j}$ 's this class includes also the GARCH(p,q) models, Bollerslev (1986); see also Taylor (1986).

An alternative approach to the above $\operatorname{ARCH}(\infty)$ model class is the class of stochastic volatility models a simple form of which is given if $\sigma_{t}$ in (1) satisfies

$$
\sigma_{t}=g\left(h_{t}\right), \quad \text { with } h_{t}=a_{0}+a_{1} h_{t-1}+e_{t},
$$

where $g(\cdot)>0$ is a known function and $\left\{e_{t}\right\}$ is an i.i.d. process independent from $\left\{\varepsilon_{t}\right\}$. For $\left|a_{1}\right|<1$ the process $h_{t}$ is strictly stationary. Notice that the heteroscedastic variation of $R_{t}$ described by $\sigma_{t}^{2}$ is driven by the unobservable latent process $\left\{h_{t}\right\}$ and not by the lagged values of the log-returns $R_{t-1}, R_{t-2}, \ldots$. Thus for this model class, the volatility function is modeled as a strictly stationary process $\left\{\sigma_{t}^{2}\right\}$ independent of the i.i.d. noise process $\left\{\varepsilon_{t}\right\}$ avoiding therefore any kind of feedback between the noise and the volatility process; cf. Shepard (1996).

Given a time series $R_{1}, R_{2}, \ldots, R_{n}$ of log-returns, one is commonly interested in the construction of point or interval estimators of the volatility function $\sigma_{t}^{2}$ and in testing hypothesis about this function. Several estimation methods have been proposed in the literature depending on the assumptions imposed on $\sigma_{t}^{2}$. They rank from parametric methods designed when $\sigma_{t}^{2}$ belongs to certain parametric classes of models to fully nonparametric methods based on weak assumptions on the function of interest. In this context, nonparametric procedures are useful not only because they lead to estimators of the underlying volatility function without imposing too restrictive assumptions but also because they are very useful for model selection and testing by means of comparing parametric and nonparametric estimates; cf. for instance Kreiss et al. (2004).

Assigning properties of estimators of $\sigma_{t}^{2}$ is usually carried out by means of asymptotic considerations where the expressions obtained for the asymptotic quantities usually depend in a complicated way on characteristics of the underlying process. This makes alternative approaches based on bootstrap 
methodology appealing. During the last decades different bootstrap methods have been proposed in the context of financial time series. Some early application of the bootstrap in financial time series mainly based on i.i.d. resampling (cf. Maddala and Li (1996) and Ruiz and Pascual (2002) for a review) are not appropriate and may lead to wrong conclusions since log-returns are not independent despite their vanishing correlation. Thus approaches to bootstrap financial time series should take into account their dependence structure or at least those aspects of this dependence structure which are important for the particular inference problem at hand.

Nonparametric methods to bootstrap time series which are based on resampling with replacement from blocks of consecutive observations can in principle be applied to financial series, and their properties to approximate the distribution of statistics of interest can be investigated provided the underlying stochastic process obeys some appropriate weak dependence, e.g., mixing conditions. For mixing properties of some commonly used time series models with applications in finance, see for instance, Carrasco and Chen (2002). Under appropriate mixing conditions, bootstrapping a series of logreturns $R_{1}, R_{2}, \ldots, R_{n}$ can be done by randomly choosing with replacement a number of $l, l=[n / b]$, blocks of $b$ consecutive values $\left\{R_{t}, R_{t+1}, \ldots, R_{t+b-1}\right\}$ from all possible $n-b$ blocks; cf. Künsch (1989), Liu and Sign (1992), Politis and Romano (1994); see also Bühlmann (2002) and Härdle et al. (2003) for an overview and the monograph by Lahiri (2003). Although such general blocking techniques preserve the dependence structure of the observations within blocks, they have not been widely used in the context of financial time series. A reason for this might be that since it is common to impose some kind of model structure in describing the behavior of financial time series, efficiency considerations make model-based bootstrap methods more attractive. Furthermore, and concerning nonparametric estimators of the volatility function $\sigma_{t}^{2}$, it is well-known that the dependence structure of the underlying process affects the limiting behavior of the estimators only through the behavior of certain finite dimensional, stationary distributions of the process. This again suggests that bootstrapping nonparametric estimators for financial time series can be successfully done without mimicking the whole and probably very complicated dependence structure of the observed series.

In Section 2 we review different methods to bootstrap financial time series, that is methods that can be applied to generate pseudo-replicates $R_{1}^{*}, R_{2}^{*}, \ldots, R_{n}^{*}$ of the observed series of log-returns. Such methods are designed in a way that mimics closely the essential dependence characteristics of the observed time series or at least those characteristics which are important for inferring consistently properties of the statistics of interest. In Section 3 we concentrate on the somewhat different problem on how to apply the bootstrap in order to infer properties of statistics based on financial time series. Such methods do not work by generating new pseudo-observations of the observed log-returns that preserves their dependence structure, but by generating pseudo-replicates of the statistic of interest. Section 4 is devoted to subsampling and self-normalization methods applied to financial data. 


\section{Resampling the Time Series of Log-Returns}

The bootstrap procedures described in this section generate replications $R_{1}^{*}, R_{2}^{*}, \ldots, R_{n}^{*}$ of the series of log-returns. Bootstrap-based inference is then provided by approximating properties of the statistics based on the original time series $R_{1}, R_{2}, \ldots, R_{n}$ by the corresponding properties of the statistics based on the bootstrap replicates $R_{1}^{*}, R_{2}^{*}, \ldots, R_{n}^{*}$.

\subsection{Parametric methods based on i.i.d. resampling of residuals}

The basic idea to bootstrap a series of log-returns when parametric assumptions on the volatility function $\sigma_{t}^{2}(\cdot)$ are imposed, is to obtain residuals using the estimated parametric model and to generate new pseudo-series of logreturns using the estimated model structure and i.i.d. resampling of residuals. More specifically, suppose that

$$
R_{t}=\sigma_{t}(\theta) \varepsilon_{t}
$$

where the function $\sigma_{t}^{2}(\theta)$ belongs to some parametric family of functions with $\theta \in \Theta$ and $\Theta$ a finite dimensional parameter space. Furthermore, $\left\{\varepsilon_{t}\right\}$ denotes a sequence of i.i.d. random variables with zero mean and unit variance.

As an example consider a $\operatorname{GARCH}(\mathrm{p}, \mathrm{q})$ specification of $\sigma_{t}^{2}(\theta)$ given by

$$
\sigma_{t}^{2}(\theta)=c+\sum_{i=1}^{p} a_{i} R_{t-i}^{2}+\sum_{j=1}^{q} b_{j} \sigma_{t-j}^{2},
$$

$\theta=\left(c, a_{1}, \ldots, a_{p}, b_{1}, \ldots, b_{q}\right)^{\prime}, c>0, a_{i} \geq 0, b_{j} \geq 0$ and $p$ and $q$ nonnegative integers. Strictly stationarity of the stochastic process $\left\{X_{t}, t \in Z\right\}$ with $E\left(X_{t}^{2}\right)<\infty$ follows if $\sum_{i=1}^{p} a_{i}+\sum_{j=1}^{q} b_{j}<1$, Bollerslev (1986); see also Bougerol and Picard (1992). Let $\left\{\widehat{\theta}=\widehat{\theta}_{n}, n \in N\right\}$ be a sequence of estimators $\widehat{\theta}=\left(\widehat{c}, \widehat{a}_{1}, \ldots, \widehat{a}_{p}, \widehat{b}_{1}, \ldots, \widehat{b}_{q}\right)^{\prime}$ of $\theta$, for instance, the commonly used conditional maximum likelihood estimator. Define standardized residuals

$$
\widehat{\varepsilon}_{t}=\frac{\widetilde{\varepsilon}_{t}-\widetilde{n}^{-1} \sum_{i} \widetilde{\varepsilon}_{i}}{\left\{\widetilde{n}^{-1} \sum_{i} \widetilde{\varepsilon}_{i}^{2}-\left(\widetilde{n}^{-1} \sum_{i} \widetilde{\varepsilon}_{i}\right)^{2}\right\}^{1 / 2}},
$$

where $\widetilde{\varepsilon}_{t}=R_{t} / \sigma_{t}(\widehat{\theta})$ are the estimated model residuals and $\widetilde{n}=n-p$. Pseudoseries of $\log$ returns following a $\operatorname{GARCH}(\mathrm{p}, \mathrm{q})$ model structure can then be generated using the equation

$$
R_{t}^{*}=\sigma_{t}(\widehat{\theta}) \varepsilon_{t}^{*}
$$

where $\sigma_{t}(\widehat{\theta})$ is the specification (6) of the conditional variance function with $\theta$ replaced by its estimator $\widehat{\theta},\left\{\varepsilon_{t}^{*}\right\}$ is an i.i.d. sequence with $\varepsilon_{t}^{*} \sim F_{n}$ and $F_{n}$ is the empirical distribution function of the $\widehat{\varepsilon}_{t}$ 's.

In most applications of such a parametric bootstrap procedure it is assumed that $\varepsilon_{t}$ has finite fourth moments, i.e., $E \varepsilon_{t}^{4}<\infty$. For instance and for $\varepsilon_{t}$ being standard Gaussian errors, Kokoszka et al. (2004) use this bootstrap method to construct confidence intervals for the autocorrelations of the 
squares of log-returns, while Miguel and Olave (1999) considered parametric bootstrap predition intervals for $\mathrm{ARCH}$ processes. The assumption of finite fourth moments is crucial for proving consistency of such a parametric bootstrap, since, loosely speaking, this assumption ensures asymptotic normality of the estimator involved with an appropriate, $\sqrt{n}$-rate of convergence. Without such a moment assumption consistency of this parametric bootstrap procedure might be questionable.

For instance, let $\hat{\theta}^{*}$ be the estimator of $\theta$ based on the pseudo-returns $R_{1}^{*}, R_{2}^{*}, \ldots, R_{n}^{*}$ and consider the problem of estimating the distribution of an appropriately rescaled version of $\widehat{\theta}-\theta$ by the corresponding distribution of the bootstrap estimator $\widehat{\theta}^{*}-\widehat{\theta}$. In such a context, consistency of the parametric bootstrap procedure depends on the limiting behavior of $\widehat{\theta}-\theta$ which in turn depends on the distribution of the i.i.d. errors $\varepsilon_{t}$. If $E\left(\varepsilon_{1}\right)^{4}<\infty$ then it has been shown by Berkes et al. (2003) that under some regularity conditions, $\sqrt{n}(\widehat{\theta}-\theta) \Rightarrow N\left(0,\left(E \varepsilon_{1}^{4}-1\right) \Sigma_{\theta}^{-1}\right)$ as $n \rightarrow \infty$, where $\Sigma_{\theta}=E\left(\sigma_{1}^{-4}(\theta) U(\theta) U^{\prime}(\theta)\right)$ and $U(\theta)$ is the $r$-dimensional vector of first derivatives of $\sigma_{1}^{2}(\theta)=\sigma_{1}^{2}(a, b, c)$ with respect to the components of $a=\left(a_{1}, \ldots, a_{p}\right), b=\left(b_{1}, \ldots, b_{q}\right)$ and $c$, evaluated at $\theta$. The asymptotic normality of $\sqrt{n}(\widehat{\theta}-\theta)$ in this case, suggests the use of the distribution of $\sqrt{n}\left(\widehat{\theta}^{*}-\widehat{\theta}\right)$ to approximate the distribution of the former statistic. However, if $E\left(\varepsilon_{1}^{4}\right)=\infty$ then the normalizing sequence of the estimation error $\widehat{\theta}-\theta$ is no more $\sqrt{n}$ and the limiting distribution of the appropriately normalized sequence $\widehat{\theta}-\theta$ differs from a Gaussian law and depends on the distribution of the squared errors $\varepsilon_{1}^{2}$; see Straumann (2005) and Hall and Yao (2003). In such situations, subsampling offers an alternative; cf. Politis et al. (1999). A subsampling based approach to infer properties of parameter estimators for GARCH processes which is asymptotically valid under general assumptions on the distribution of the errors $\varepsilon_{t}$ has been proposed by Hall and Yao (2003); see Section 4 for details.

\subsection{Nonparametric methods based on i.i.d. resampling of residuals}

If instead of a parametric structure for $\sigma(\cdot)$ the general nonparametric model

$$
R_{t}=\sigma\left(R_{t-1}, R_{t-2}, \ldots, R_{t-p}\right) \varepsilon_{t}
$$

is assumed, then the volatility function $\sigma^{2}(\cdot)$ can be estimated in a nonparametric way and pseudo-series of log-returns can be generated using the estimated volatility function and i.i.d. resampling of estimated residulas. Meaningful statistical inference for $\sigma^{2}(\cdot)$ in a nonparametric context, requires however, that the discrete time Markov process $\left\{R_{t}\right\}$ following (7) satisfies some stability and ergodicity conditions which can be achieved by imposing some restrictions on the distribution of the errors $\varepsilon_{t}$ and on the function $\sigma: R^{p} \rightarrow(0, \infty)$. For instance, when $p=1,\left\{R_{t}\right\}$ is geometrically ergodic if the density $f_{\varepsilon}$ of $\varepsilon$ is absolutely continuous and positive everywhere, $\inf _{x \in R} \sigma(x)>0, \sigma$ is bounded over bounded sets and satisfies $\lim \sup _{|x| \rightarrow \infty} E\left|\sigma(x) \varepsilon_{1}\right| /|x|<1$; cf. Franke et al. (2002b).

Let $\widehat{\sigma}^{2}\left(x_{1}, \ldots, x_{p}\right)$ be a nonparametric estimator of $\sigma^{2}\left(x_{1}, \ldots, x_{p}\right)=$ $E\left(R_{t}^{2} \mid R_{t-1}=x_{1}, \ldots, R_{t-p}=x_{p}\right)$ and define standardized residuals 


$$
\widehat{\varepsilon}_{t}=\frac{\widetilde{\varepsilon}_{t}-\widetilde{n}^{-1} \sum_{i} \widetilde{\varepsilon}_{i}}{\left\{\widetilde{n}^{-1} \sum_{i} \widetilde{\varepsilon}_{i}^{2}-\left(\widetilde{n}^{-1} \sum_{i} \widetilde{\varepsilon}_{i}\right)^{2}\right\}^{1 / 2}},
$$

where $\widetilde{\varepsilon}_{t}=R_{t} / \widehat{\sigma}\left(R_{t-1}, R_{t-2}, \ldots, R_{t-p}\right), t=p+1, p+2, \ldots, n$ and $\widetilde{n}=n-p$. Pseudo-series of log returns can then be generated as

$$
R_{t}^{*}=\widehat{\sigma}\left(R_{t-1}^{*}, \ldots, R_{t-p}^{*}\right) \varepsilon_{t}^{*},
$$

where the $\varepsilon_{t}^{*}$ 's are i.i.d. random variables with $\varepsilon_{t}^{*} \sim F_{n}$ and $F_{n}$ is the empirical distribution function of the $\widehat{\varepsilon}_{t}$; cf. Franke et al. (2002a).

To fix ideas, let $p=1$ and consider the following nonparametric estimator of the volatility function

$$
\widehat{\sigma}_{h}^{2}(x)=\frac{1}{\widehat{f}_{R_{t}}(x)} \frac{1}{(n-1)} \sum_{t=1}^{n-1} K_{h}\left(x-R_{t}\right)\left(R_{t+1}-\bar{R}_{n}\right)^{2},
$$

where $h$ is a smoothing bandwidth, $K$ a smoothing kernel and $\bar{R}_{n}=$ $n^{-1} \sum_{t=1}^{n} R_{t}$. Notice that centering by the sample mean $\bar{R}_{n}$ is used since it is assumed that $\mu_{t}=0$. If the conditional mean function $\mu_{t}$ is not constant and $\mu_{t}=m\left(R_{t-1}\right)$ with $m(\cdot)$ some smooth function, then $\bar{R}_{n}$ should be replaced by a nonparametric estimator $\widehat{m}_{h}\left(R_{t-1}\right)$ of the conditional mean function $m\left(R_{t-1}\right)=E\left(R_{t} \mid R_{t-1}\right)$; see Fan and Yao (1998) for details and for a comparison of different estimators of the volatility function.

The bootstrap procedure described above can be applied to approximate the distribution of random variables like $\sqrt{n h}\left(\widehat{\sigma}_{h}^{2}(x)-\sigma^{2}(x)\right)$ and $\sup _{x \in[a, b]}\left|\widehat{\sigma}_{h}^{2}(x)-\sigma^{2}(x)\right|$, for some $a<b$, or of some standardized versions thereof. Franke et al (2002b) established under certain regularity conditions absolute regularity and geometric ergodicity of the bootstrap process $\left\{R_{t}^{*}, t \in Z\right\}$ and applied these results to show validity of the corresponding nonparametric bootstrap procedure in approximating the distribution of supremum type statistics of the conditional mean estimator. Franke et al. (2000a) consider applications to pointwise statistics while Franke et al. (2004) investigated properties of such a bootstrap procedure for the construction of confidence bands for the volatility function.

Notice that an inherent problem common in applications of the bootstrap to estimate the distribution of nonparametric estimators is how to deal with the bias. In particular, and decomposing the statistic of interest in a stochastic and in a bias term, i.e., writing

$$
\sqrt{n h}\left(\widehat{\sigma}_{h}^{2}(x)-\sigma^{2}(x)\right)=\sqrt{n h}\left(\widehat{\sigma}_{h}^{2}(x)-E\left(\widehat{\sigma}_{h}^{2}(x)\right)+\sqrt{n h}\left(E\left(\widehat{\sigma}_{h}^{2}(x)\right)-\sigma^{2}(x)\right),\right.
$$

makes it clear that if one is interested in applying the bootstrap in order to approximate the distribution of $\sqrt{n h}\left(\widehat{\sigma}_{h}^{2}(x)-\sigma^{2}(x)\right)$, then such a bootstrap procedure should approximate correctly the behavior of both terms on the right hand side of the above decomposition. In this context correct estimation of the bias term requires a kind of oversmoothing, i.e., the use of an estimator $\widehat{\sigma}_{g}^{2}(x)$ to generate the pseudo-variables $R_{t}^{*}$ 's in (8) for which the bandwidth $g$ satisfies $g>h$ and $h / g \rightarrow 0$ as $n \rightarrow \infty$. The bootstrap statistic used to approximate the distribution of $\sqrt{n h}\left(\widehat{\sigma}_{h}^{2}(x)-\sigma^{2}(x)\right)$ is then given by 
$\sqrt{n h}\left(\widehat{\sigma}_{h}^{*^{2}}(x)-\sigma_{g}^{2}(x)\right)$ where $\widehat{\sigma}_{h}^{*^{2}}(x)$ is the estimator (9) with $R_{t}$ replaced by the bootstrap pseudo-variables $R_{t}^{*}$ and $\bar{R}_{n}$ by $\bar{R}_{n}^{*}=n^{-1} \sum_{t=1}^{n} R_{t}^{*}$. An alternative to such a bootstrap estimation of the bias would be explicit bias correction; see Hall(1992) for a discussion of this issue in the context of independent data.

Although the above nonparametric bootstrap procedure can be successfully applied to estimate the distribution of the nonparametric estimators of interest, it fails if the process of log-returns does not follow model (7). The reason for this is that in this case the stationary distribution of the bootstrap variables $\left(R_{t}^{*}, R_{t-1}^{*}, \ldots, R_{t-p}^{*}\right)$ does not converge to the desired stationary distribution of $\left(R_{t}, R_{t-1}, \ldots, R_{t-p}\right)$. In fact and for $\mathbf{R}_{t-1, t-p}=$ $\left(R_{t-1}, R_{t-2}, \ldots, R_{t-p}\right)$, we expect is such a case that the conditional distribution function $P\left(R_{t}^{*} \leq \cdot \mid \mathbf{R}_{t-1, t-p}^{*}=\mathbf{x}\right)$ of the bootstrap process will behave asymptotically like $F_{U_{t}}(\cdot / \sigma(\mathbf{x}))$ where $F_{U_{t}}$ denotes the stationary distribution function of $U_{t}=R_{t} / \sigma(\mathbf{x})$ and $\sigma(\mathbf{x})=\sqrt{\sigma^{2}(\mathbf{x})}$ with $\sigma^{2}(\mathbf{x})=E\left(R_{t}^{2} \mid R_{t-i}=\right.$ $\left.x_{i}, i=1,2, \ldots, p\right)$. This distribution differs however from the conditional distribution function $P\left(R_{t} \leq \cdot \mid \mathbf{R}_{t-1, t-p}=\mathbf{x}\right)$ of the underlying process if model (7) is not correct.

\subsection{Markovian bootstrap}

Model (7) is a special case of a more general $p$-th order Markovian process, that is a process $\left\{R_{t}, t \in Z\right\}$ which satisfies

$$
P\left(R_{t} \in A \mid \sigma\left(R_{s}, s<t\right)\right)=P\left(R_{t} \in A \mid R_{t-1}, R_{t-2}, \ldots, R_{t-p}\right),
$$

for all $A \in \mathcal{B}(R)$ and all $t \in Z$. Suppose that such a model describes the behavior of log-returns and denote by $F_{R_{t} \mid \mathbf{R}_{t-1, t-p}}(\cdot \mid \mathbf{x})=P\left(R_{t} \leq y \mid R_{t-i}=\right.$ $\left.x_{i}, i=1,2, \ldots, p\right)$ the one-step transition distribution function. Assume that the corresponding conditional probability measure possesses a density with respect to Lebesgue measure which we denote by $f_{R_{t} \mid \mathbf{R}_{t-1, t-p}}(\cdot \mid \mathbf{x})$. Notice that model (7) is a specific Markov process with one-step transition distribution function given by $F_{R_{t} \mid \mathbf{R}_{t-1, t-p}}(y \mid \mathbf{x})=F_{\varepsilon}(y / \sigma(\mathbf{x}))$ where $F_{\varepsilon}$ is the distribution function of the error $\varepsilon_{1}$.

Imposing conditions on $F_{R_{t} \mid \mathbf{R}_{t-1, t-p}}(\cdot)$ which ensure stationarity and geometric ergodicity of the associated Markov chain, bootstrap replicates of log-returns can be generated using the Markovian model structure without specifying its functional form. Rajarshi (1990) proposed such a bootstrap approach based on a nonparametric estimator $\widehat{f}_{R_{t} \mid \mathbf{R}_{t-1, t-p}}(\cdot \mid \mathbf{x})$ of the one step transition density $f_{R_{t} \mid \mathbf{R}_{t-1, t-p}}(\cdot \mid \mathbf{x})$. Such a nonparametric estimator is for instance given by

$$
\widehat{f}_{R_{t} \mid \mathbf{R}_{t-1, t-p}}(y \mid \mathbf{x})=\sum_{t=p+1}^{n} K_{b}\left((y, \mathbf{x})-\mathbf{R}_{t, t-p}\right) / \sum_{t=p+1}^{n} K_{b}\left(\mathbf{x}-\mathbf{R}_{t-1, t-p}\right),
$$

where $b$ is the bandwidth used to estimate the stationary densities of interest. New series of pseudo-replications can then be generated as

$$
R_{t}^{*} \sim \widehat{f}_{R_{t} \mid \mathbf{R}_{t-1, t-p}}\left(\cdot \mid R_{t-1}^{*}, R_{t-2}^{*}, \ldots, R_{t-p}^{*}\right) .
$$


A different approach which does not require explicit nonparametric estimation and resamples directly the original series of log-returns in an appropriate way preserving their Markovian dependence structure, has been proposed by Paparoditis and Politis (2001a). Given a series $R_{t-p}^{*}, R_{t-p+1}^{*}, \ldots, R_{t-1}^{*}$ of pseudo log-returns, their approach works by generating a new pseudo-variable $R_{t}^{*}$ as

$$
R_{t}^{*}=R_{J},
$$

where $J$ is a discrete random variable taking values in the set $\{p+1, p+$ $2, \ldots, n\}$ with

$$
P(J=s)=\frac{W_{b}\left(\mathbf{R}_{t-1, t-p}^{*}-\mathbf{R}_{s-1, s-p}\right)}{\sum_{l=p+1}^{n} W_{b}\left(\mathbf{R}_{t-1, t-p}^{*}-\mathbf{R}_{l-1, l-p}\right)}
$$

for $s \in\{p+1, p+2, \ldots, n\}$, where $\mathbf{R}_{t-1, t-p}^{*}=\left(R_{t-p}^{*}, R_{t-p+1}^{*}, \ldots, R_{t-1}^{*}\right)$. Here $W_{b}(\cdot)=b^{-p} W(\cdot / p)$, where $b$ is the so-called resampling width and $W(\cdot)$ a $p$-dimensional, nonnegative and symmetric resampling kernel with mean zero. Notice that this procedure resamples the observed log-returns in a way according to which the probability of $R_{s}$ being selected is higher the closer is its preceding segment $\left(R_{s-1}, R_{s-2}, \ldots, R_{s-p}\right)$ to the last generated bootstrap segment $\left(R_{t-1}^{*}, R_{t-2}^{*}, \ldots, R_{t-p}^{*}\right)$.

Properties of Markovian bootstrap procedures have been investigated by Rajarshi (1990) and Paparoditis and Politis (2001a); see also Horowitz (2003). Applications of such a bootstrap procedure in order to approximate the distribution of nonparametric conditional moment estimators and for constructing pointwise confidence intervals have been investigated by Paparoditis and Politis (2002). Notice that in approximating correctly the bias of the nonparametric estimators involved, a kind of oversmoothing condition is needed here as well leading to some restrictions on the behavior of the resampling width $b$ compared to the smoothing bandwidth $h$. In particular, $b$ should satisfy $b>h$ and $b / h \rightarrow 0$ as $n \rightarrow \infty$.

We stress here the fact that for the kind of nonparametric estimators discussed in this paper, the range of applicability of the above Markovian procedures goes far beyond the Markov process class. This is due to the fact that the Markovian resampling schemes described above mimics correctly the $(p+1)$-dimensional stationary distribution of $\left(R_{t}, R_{t-1}, \ldots, R_{t-p}\right)$ even if the underlying process is not Markov. This property suffices to establish consistency of the above Markovian bootstrap procedures applying to estimate the distribution of the nonparametric estimators of interest for a very broad class of stochastic processes; see Paparoditis and Politis (2002) for details.

\section{Resampling Statistics Based on the Time Series of Log-Returns}

In the context of dependent data it is possible in certain situations to apply the bootstrap to some statistics of interest without generating new time series of pseudo-observations that preserve the dependence structure of the observed time series. Such applications resample directly the statistic of interest in a 
way which mimics correctly those characteristics of the dependence structure of the underlying process which are essential for the random behavior of the statistic of interest.

Suppose for instance, that we are interested in approximating the poinwise distribution of $\sqrt{n h}\left(\widehat{\sigma}_{h}^{2}(x)-\sigma^{2}(x)\right)$ by means of the bootstrap. As it has been already stressed, for a bootstrap procedure to be successful in approximating correctly the (limiting) distribution of this statistic, it is not necessary to imitate the whole and probably very complicated dependence structure of the underlying process of log-returns. For this, it suffices to mimic correctly the $(p+1)$-dimensional stationary distribution of $\left(R_{t}, R_{t-1}, \ldots, R_{t-p}\right)$. This is a consequence of the basic fact that the asymptotic distribution of nonparametric estimators does not reflect the dependence structure of the underlying process beyond the $(p+1)$-dimensional structure; cf. Robinson (1983). Hart (1995) called this the whitening by windowing effect. This basic observation has motivated the development of bootstrap procedures that generate pseudoreplicates $\left(R_{t}^{*}, R_{t-1}^{*}, \ldots, R_{t-p}^{*}\right)$ of $\left(R_{t}, R_{t-1}, \ldots, R_{t-p}\right)$ in a way that mimic correctly the joint distribution of the last random vector, without generating new pseudo-series of log-returns.

\subsection{Regression Bootstrap}

The regression bootstrap is a nonparametric bootstrap procedure which generates replicates of the pairs $\left\{\left(R_{t}, \mathbf{R}_{t-1, t-p}\right), t=p+1, p+2, \ldots, n\right\}$ denoted by $\left\{\left(R_{t}^{*}, \mathbf{R}_{t-1, t-p}\right), t=p+1, p+2, \ldots, n\right\}$ by using a fixed design, heteroscedastic regression model with errors obtained by i.i.d. resampling from estimated model residuals; cf. Franke et al. (2002a). Notice that in this resampling scheme only the random variable $R_{t}$ is bootstrapped while $\mathbf{R}_{t-1, t-p}$ is treated as a (conditionally) fixed design. In particular, the bootstrap variables $R_{t}^{*}$ are generated using the equation

$$
R_{t}^{*}=\widehat{\sigma}_{g}\left(R_{t-1}, R_{t-2}, \ldots, R_{t-p}\right) \varepsilon_{t}^{*},
$$

where the $\varepsilon_{t}^{*}$ are independent random variables such that $\varepsilon_{t}^{*} \sim F_{n}$ and $F_{n}$ is the empirical distribution function of the estimated errors $\widehat{\varepsilon}_{t}$ given in Section 2.2. Notice that the random variables $R_{t}^{*}$ are (conditional on the observed series) independent with $E^{*}\left(R_{t}^{*}\right)=0$ and $\operatorname{Var}^{*}\left(R_{t}^{*}\right)=\widehat{\sigma}_{g}^{2}\left(R_{t-1}, R_{t-2}, \ldots, R_{t-p}\right)$. Thus the dependence structure of the series of log returns is not preserved by this bootstrap method. The distribution of $\sqrt{n h}\left(\widehat{\sigma}_{h}^{2}(\mathbf{x})-\sigma^{2}(\mathbf{x})\right)$ can now be approximated by that of $\sqrt{n h}\left(\widehat{\sigma}_{h}^{*}(\mathbf{x})-\widehat{\sigma}_{g}^{2}(\mathbf{x})\right)$, where, for $p=1$,

$$
\widehat{\sigma}_{h}^{*^{2}}(x)=\frac{1}{\widehat{f}_{R_{t}}(x)} \frac{1}{(n-1)} \sum_{t=1}^{n-1} K_{h}\left(x-R_{t}\right)\left(R_{t+1}^{*}-\bar{R}_{n}^{*}\right)^{2},
$$

and $\bar{R}_{n}^{*}=n^{-1} \sum_{t=1}^{n} R_{t}^{*}$. The bandwidth $g$ used in (10) can be chosen so that the above bootstrap procedure estimates also correctly the bias term $\sqrt{n h}\left(E\left(\widehat{\sigma}_{h}^{2}(x)\right)-\sigma^{2}(x)\right)$ of the nonparametric volatility function estimator. For this an oversmoothing type condition should be satisfied, i.e., $g>h$ with $h / g \rightarrow 0$ as $n \rightarrow \infty$; cf. Franke et al. (2002a). 


\subsection{Wild bootstrap}

The wild bootstrap methodology can be also applied in the context of financial time series to estimate the distribution of some nonparametric estimators of interest; see Franke et al. (2002a) and Kreiss (2000). To fix ideas, let $p=1$ and consider the centered log-returns $Y_{t}=R_{t}-\bar{R}_{n}, t=1,2, \ldots, n$. Let further $\eta_{t}$, $t=1,2, \ldots, n$ be a sequence of independent, identically distributed random variables satisfying $E\left(\eta_{t}\right)=0, E\left(\eta_{t}^{2}\right)=1$. For higher order performance the distribution of $\eta_{t}$ is often chosen such that additionally the condition $E\left(\eta_{t}^{3}\right)=1$ is satisfied; cf. Mammen (1992) for a discussion.

The wild bootstrap works by generating pairs $\left\{\left(Y_{t+1}^{*}, R_{t}\right), t=1,2, \ldots, n-\right.$ 1) where

$$
Y_{t+1}^{*}=\widehat{\sigma}_{g}^{2}\left(R_{t}\right)+\varepsilon_{t+1}^{*},
$$

and

$$
\varepsilon_{t+1}^{*}=\left[Y_{t+1}^{2}-\widehat{\sigma}_{h}^{2}\left(R_{t}\right)\right] \cdot \eta_{t+1}
$$

Notice that $E^{*}\left(Y_{t+1}^{*}\right)=\widehat{\sigma}_{g}^{2}\left(R_{t}\right)$ and $\operatorname{Var}^{*}\left(Y_{t+1}^{*}\right)^{2}=\left(Y_{t+1}^{2}-\widehat{\sigma}_{h}^{2}\left(R_{t}\right)\right)^{2}$. Furthermore, the bootstrap random variables $Y_{t+1}^{*}$ are (conditional on the observed sample) independent, i.e., the dependence structure of the observed time series is not preserved by this bootstrap scheme. In fact, in the bootstrap world the $Y_{t+1}^{*}$ 's are generated according to a fixed design nonparametric regression model with mean $\widehat{\sigma}_{g}^{2}\left(R_{t}\right)$ and variance $\operatorname{Var}^{*}\left(\varepsilon_{t+1}^{*}\right)$. Now, to approximate the distribution of $\sqrt{n h}\left(\widehat{\sigma}_{h}^{2}(x)-\sigma^{2}(x)\right)$ the wild-bootstrap statistic $\sqrt{n h}\left(\widehat{\sigma}_{h}^{*}(x)-\sigma_{g}^{2}(x)\right)$ can be used, where

$$
\widehat{\sigma}_{h}^{*^{2}}(x)=\frac{1}{\widehat{f}_{R_{t}}(x)} \frac{1}{(n-1)} \sum_{t=1}^{n-1} K_{h}\left(x-R_{t}\right)\left(Y_{t+1}^{*}\right)^{2} .
$$

Notice that in order to capture correctly also the bias term of the above nonparametric estimation, and similar to all bootstrap approaches discussed so far, an oversmoothing type condition is needed by the wild bootstrap as well; see Franke et al. (2002a) and Kreiss (2000).

Although the wild bootstrap does not preserve the dependence structure of the observed series, it resamples correctly the distribution of the nonparametric statistics of interest. This bootstrap scheme is robust against model misspecifications, at least as far as the estimation of the distribution of pointwise statistics like $\sqrt{n h}\left(\widehat{\sigma}_{h}^{2}(\mathbf{x})-\sigma^{2}(\mathbf{x})\right)$ is concerned; cf. Kreiss (2000). Neumann and Kreiss (1998) applied a wild bootstrap to the construction of uniform confidence bands for the conditional mean based on supremum type statistics and using strong approximation results under the assumption of a Markovian model. Kreiss (2000) considered the problem of estimating by means of a wild bootstrap procedure the distribution of the sup-distance involved in the construction of simultaneous confidence intervals for the volatility function. His approach is also based on the assumption that the underlying process obeys a Markovian dependence structure. 


\subsection{Local bootstrap}

Another simple and totally model free way to bootstrap nonparametric estimators in time series is the so-called local bootstrap; see Shi (1991) for the case of i.i.d. data and Paparoditis and Politis (2000) for the case of dependent data. This bootstrap method generates replicates $\left\{\left(R_{t}^{*}, \mathbf{R}_{t-1, t-p}\right) ; t=\right.$ $p+1, p+2, \ldots, n\}$ of the observed pairs $\left\{\left(R_{t}, \mathbf{R}_{t-1, t-p}\right) ; t=p+1, p+2, \ldots, n\right\}$ by correctly imitating the conditional distribution $F_{R_{t}, \mathbf{R}_{t-1, t-p}}(\cdot \mid \mathbf{x})$ of the $\log$ returns. In contrast to the regression bootstrap, the local bootstrap resamples the observed values $R_{t}$ by giving more resampling weights to the values $R_{s}$ for which $\mathbf{R}_{s-1, s-p}$ is close to $\mathbf{R}_{t-1, t-p}$.

For $p=1$ and for the problem of estimating the distribution of the nonparametric estimator (9) of the conditional variance function, this procedure can be described as follows. Let $p=1$ and $Y_{t}=R_{t}-\bar{R}_{n}, t=1,2, \ldots, n$. Bootstrap replicates $\left\{\left(Y_{t+1}^{*}, R_{t}\right), t=1,2, \ldots, n-1\right\}$ of the pairs $\left\{\left(Y_{t+1}, R_{t}\right), t=\right.$ $1,2, \ldots, n-1\}$ are then generated so that the bootstrap pseudo-variable $Y_{t+1}^{*}$ satisfies

$$
P\left(Y_{t+1}^{*}=Y_{s+1} \mid R_{t}\right)=\frac{W_{b}\left(R_{t}-R_{s}\right)}{\sum_{l=1}^{n-1} W_{b}\left(R_{t}-R_{l}\right)} .
$$

Here $b$ is the so-called resampling width which determines the neighborhood from which replicates of $Y_{t+1}$ are selected and $W(\cdot)$ a resampling kernel. It is easily seen that

$$
\left.E^{*}\left(\left(Y_{t+1}^{*}\right)^{2} \mid R_{t}=x\right)=\frac{1}{\widehat{f}_{R_{t}, b}(x)} \frac{1}{(n-1)} \sum_{t=1}^{n-1} W_{b}\left(x-R_{t}\right)\left(R_{t+1}-\bar{R}_{n}\right)\right)^{2},
$$

i.e., $E^{*}\left(\left(Y_{t+1}^{*}\right)^{2} \mid R_{t}=x\right)=\widehat{\sigma}_{b}^{2}(x)$. This suggests that the distribution of $\sqrt{n h}\left(\widehat{\sigma}_{h}^{2}(x)-\sigma^{2}(x)\right)$ can be approximated by that of $\sqrt{n h}\left(\widehat{\sigma}_{h}^{*^{2}}(x)-\widehat{\sigma}_{b}^{2}(x)\right)$ where

$$
\widehat{\sigma}_{h}^{*^{2}}(x)=\frac{1}{\widehat{f}_{R_{t}, h}(x)} \frac{1}{(n-1)} \sum_{t=1}^{n-1} K_{h}\left(x-R_{t}\right)\left(Y_{t+1}^{*}\right)^{2} .
$$

Consistency properties of such a bootstrap procedure for estimating the distribution of pointwise statistics based on nonparametric estimators of conditional moments for time series data have been established by Paparoditis and Politis (2000). Ango Nze et al. (2002) used such a procedure to estimate the distribution of nonparametric moment estimators under weak dependent assumptions (see Doukhan and Louchichi (1999)).

\section{Subsampling and Self-Normalization}

Subsampling for dependent data is a method valid in extreme generality. To define it, let $\hat{\theta}_{n}=\hat{\theta}_{n}\left(R_{1}, \ldots, R_{n}\right)$ be an arbitrary statistic that is consistent for a general parameter $\theta$ at rate $a_{n}$, i.e., for large $n$, the law of $a_{n}\left(\hat{\theta}_{n}-\theta\right)$ tends to some well-defined asymptotic distribution $J$. The rate $a_{n}$ does not have to equal $\sqrt{n}$, and the distribution $J$ does not have to be normal; we do not even need to know its shape, just that it exists. Let $\hat{\theta}_{i, b}=\hat{\theta}_{b}\left(R_{i}, \ldots, R_{i+b-1}\right)$ be the 
subsample value of the statistic computed from the $i$ th block of length $b$. The subsampling estimator of $J$ is $\hat{J}_{b, S U B}$ defined as the empirical distribution of the normalized (and centered) subsample values $a_{b}\left(\hat{\theta}_{i, b}-\hat{\theta}_{n}\right)$ for $i=1, \ldots, q$ where $q=n-b+1$.

The asymptotic consistency of $\hat{J}_{b, S U B}$ for general statistics under very weak conditions was shown in Politis and Romano (1994); consequently, confidence intervals and/or tests for $\theta$ can immediately be formed using the quantiles of $\hat{J}_{b, S U B}$ instead of the quantiles of the (unknown) $J$. Notice that if only a variance estimator for $a_{n} \hat{\theta}_{n}$ is sought, it can be constructed by the sample variance of the normalized subsample values $a_{b} \hat{\theta}_{i, b}$ for $i=1, \ldots, q$; consistency of the subsampling estimator of variance was shown by Carlstein (1986) under some uniform integrability conditions.

In addition to the usual requirement $b \rightarrow \infty$ as $n \rightarrow \infty$ but with $b / n \rightarrow$ 0 , the conditions of Politis and Romano (1994) boil down to:

C1. The series $\left\{R_{t}\right\}$ is strictly stationary.

C2. The series $\left\{R_{t}\right\}$ is strong mixing.

C3. The rate $a_{n}$ is known.

Notice that condition C3 is important for the practical construction of confidence intervals and tests, not for the consistency of subsampling as a method.

Although Conditions C1-C3 are quite weak, they manage to exclude a number of interesting settings pertaining to financial time series since the latter are often plagued by heteroscedasticity, long-range dependence, and heavy tails. Fortunately, all the above conditions can still be relaxed. A review of non-standard conditions for which (variations of) the block bootstrap are consistent can be found in Politis (2003) together with a discussion on the important issue of block size choice; see also the monograph by Lahiri (2003). Condition $\mathrm{C} 1$ can easily be relaxed to just asymptotic stationarity as enjoyed, for example, by Markov processes that are generated with an arbitrary (nonequilibrium) start-up distribution; see Politis et al. (1999, Ch. 4). The strong mixing condition $\mathrm{C} 2$ has been recently relaxed to the weak dependence condition of Doukhan and Louhichi (1999); see Ango Nze et al. (2003). Finally, condition C3 was relaxed since Bertail et al. (1999) showed how to construct subsampling-based estimators of the rate $a_{n}$ that can be employed for the construction of confidence intervals and tests.

The above can be seen as small improvements/perturbations on the original Conditions C1-C3. We now describe in more detail how to address major break-downs of those conditions using two broad techniques: (a) examination of the time series of subsample values $\hat{\theta}_{i, b}$ for $i=1, \ldots, q$, and (b) the idea of self-normalization.

To elaborate on using technique (a) in order to relax Condition C2, consider the familiar situation of a unit-root test such as the set-up in Paparoditis and Politis (2003). Under the unit-root hypothesis the data are not strong mixing, not even weakly dependent; however, the subsample values of the Phillips-Perron (say) unit-root statistic are weakly dependent and thereby the validity of subsampling is preserved; see Politis et al. (1999, Theorem 12.2.1), Politis et al. (2004), and Romano and Wolf (2001). 
The same idea can be applied to relax Condition C1. For example, consider a series that is not strictly stationary, e.g., heteroskedastic. If the subsample values of the pertinent statistic have distributions that converge to the limit $J$ in a uniform way, then subsampling remains consistent; see Politis et al. (1997). An important example is least squares regression with dependent errors and/or covariates; subsampling is shown to work here under conditions without assuming a stationary or homoskedastic error structure (Politis et al. 1997, Theorem 3.4).

The idea of self-normalization is closely related to that of studentization. To describe it, we now focus on a particular example that may defy Condition C3: assume Condition $\mathrm{C} 1$, and consider the simple case where the statistic of interest $\hat{\theta}_{n}=n^{-1} \sum_{t=1}^{n} R_{t}$ is the sample mean which is an estimator of the expected value $\theta=E\left(R_{t}\right)$ (assumed to be finite). As previously mentioned, the rate $a_{n}$ is not necessarily $\sqrt{n}$. Two major avenues resulting in a rate that is less than $\sqrt{n}$ are heavy tailed data, and/or long-range dependence.

To fix ideas, suppose that $a_{n}=n^{\alpha}$ for some unknown $\alpha \in(0,1]$. A succesful self-normalization entails constructing a positive statistic, say $\hat{\zeta}_{n}>0$, that converges to some limit distribution $Q$ at a rate explicitly related to the unknown $\alpha$. For example, consider the case where $n^{\alpha-\delta} \hat{\zeta}_{n}$ has limit distribution $Q$ for some known value $\delta>0$. Then, subsampling can be applied to the self-normalized quantity $\left(\hat{\theta}_{n}-\theta\right) / \hat{\zeta}_{n}$ that converges to a well-defined distribution at the known rate $n^{\delta}$. Strictly speaking, the joint convergence of $\left(n^{\alpha}\left(\hat{\theta}_{n}-\theta\right), n^{\alpha-\delta} \hat{\zeta}_{n}\right)$ to the pair $(J, Q)$ is required; see e.g. Politis et al. (1999, Theorem 11.3.1) for a precise statement.

Typically, the search for a suitable $\hat{\zeta}_{n}$ starts with an estimated standard deviation for $\hat{\theta}_{n}$-hence the connection to studentization. The self-normalization method for subsampling was first used by Hall et al. (1998) in the context of a long-range dependent data. Hall and Yao (2003) also employ selfnormalization in connection with bootstrap with smaller resample size on the residuals of heavy tailed GARCH processes. As is well-known, the bootstrap with smaller resample size is closely related to subsampling in the i.i.d. case; see Politis et al. (1999, Ch. 2.3). The case of self-normalized subsampling for heavy tailed time series was addressed by McElroy and Politis (2002), and Kokoszka and Wolf (2004). McElroy and Politis (2006) is the only paper todate that achieves a self-normalization in a setting exhibiting both heavy-tails and long-range dependence.

\section{References}

1. Ango Nze P, Bühlmann P and Doukhan P (2002) Weak dependence beyond mixing and asymptotics for nonparametric regression. Annals of Statistics 30:397-430

2. Ango Nze P, Dupoiron S and Rios R (2003) Subsampling under weak dependence conditions, Tech. Report DT2003-42, CREST, Paris

3. Berkes I, Horváth L and Kokoszka P (2003) GARCH processes: structure and estimation. Bernoulli 9:201-228 
4. Bertail P, Politis D N and Romano J P (1999) On subsampling estimators with unknown rate of convergence, Journal of the American Statistical Association 94: 569-579.

5. Bollerslev T (1986) Generalized autoregressive conditional heteroscedasticity. Journal of Econometrics 31:307-327

6. Bougerol P and Picard N (1992) Stationarity of GARCH processes and of some nonnegative time series. Journal of Econometrics 52:115-127

7. Bühlmann P (2002) Bootstrap for time series. Statistical Science 17: 52-72

8. Carlstein E (1986) The use of subseries values for estimating the variance of a general statistic from a stationary time series. Annals of Statistics 14: 1171-1179

9. Carrasco M and Chen X (2002) Mixing and moment properties of various GARCH and stochastic volatility models. Econometric Theory 18:17-39

10. Doukhan P and Louhichi S (1999) A new weak dependence condition and applications to moment inequalities. Stochastic Processes and its Applications 84: 313-342

11. Engle R (1982) Autoregressive conditional heteroskedasticity with estimates of the variance of U.K. inflation. Econometrica 50:987-1008

12. Fan J and Yao Q (1998) Efficient estimation of conditional variance functions in stochastic regression. Biometrika 85:645-660

13. Franke J, Kreiss J P and Mammen E (2002a) Bootstrap of kernel smoothing in nonlinear time series. Bernoulli 8:1-37

14. Franke J, Kreiss J P, Mammen E and Neumann M H (2002b) Properties of the nonparametric autoregressive bootstrap. Journal of Time Series Analysis 23:555-585

15. Franke J, Neumann M H and Stockis J P (2004) Bootstrapping nonparametric estimators of the volatility function. Journal of Econometrics 118:189-218.

16. Giraitis L, Kokoszka P and Leipus R (2000) Stationary ARCH models: Dependence structure and central limit theorem. Econometric Theory 16:3-22.

17. Hall P (1992) The bootstrap and Edgeworth expansion. Springer Verlag, New York

18. Hall P, Jing B-Y and Lahiri S N (1998) On the sampling window method for long-range dependent data. Statistica Sinica 8:1189-1204

19. Hall P and Yao Q (2003) Inference in ARCH and GARCH models with heavytailed errors. Econometrica 71:285-317

20. Härdle W, Horowitz J and Kreiss J P (2003) Bootstrap for Time Series. International Statistical Review 71:435-459

21. Hart, J D (1995) Some automated methods of smoothing time-dependent data. Journal of Nonparametric Statistics 6:115-142

22. Horowitz J L (2003) Bootstrap methods for Markov processes. Econometrica 71:1049-1082

23. Kazakevičius V and Leipus R (2002) On stationarity in the $\mathrm{ARCH}(\infty)$ model. Econometric Theory 18:1-16

24. Kokoszka P, Teyssiére G and Zhang A (2004) Confidence intervals for the autocorrelations of the squares of GARCH sequences. In: M. Bubak et al. (eds) ICCS 2004. Springer, Berlin, p. 827-834

25. Kokoszka P and Wolf M (2004) Subsampling the mean of heavy-tailed dependent observations. Journal of Time Series Analysis 25:217-234

26. Kreiss J P (2000) Nonparametric estimation and bootstrap for financial time series. In: Chan W S, Li W K and Tong H (eds). Statistics and Finance: An Interface. London, Imperial College Press.

27. Kreiss J P, Neumann M H and Q. Yao (2004) Bootstrap tests for simple structures in nonparametric time series regression. Preprint 
28. Künsch H R (1989) The jackknife and the bootstrap for general stationary observations. Annals of Statistics 17:1217-1241

29. Lahiri S N (2003) Resampling methods for dependent data. Springer, New York

30. Liu R and Singh K (1992) Moving blocks jackknife and bootstrap capture weak dependence. In: LePage R and Billard L (eds) Exploring the limits of the bootstrap. Wiley, New York, p. 225-248

31. Maddala G S and Li H (1996) Bootstrap based tests in financial models. In: Maddala G S and Rao C R (eds). Handbook of Statistics, vol. 14, Amsterdam, Elsevier, p. 463-488

32. Mammen E. (1992) When does the bootstrap work? Asymptotic results and simulations. Springer Lecture Notes in Statistics 77. Singer Verlag, Heidelberg

33. McElroy T and Politis D N (2002) Robust inference for the mean in the presence of serial correlation and heavy tailed distributions. Econometric Theory 18: 1019-1039

34. McElroy T and Politis D N (2006) Self-Normalization for heavy-tailed time series with long memory. Statistica Sinica, to appear

35. Miquel J A and Olave P (1999) Bootstrapping forecast intervals in ARCH models. Test 8:345-364

36. Neumann M H and Kreiss J-P (1998) Regression type inference in nonparametric autoregression. Annals of Statistics 26:1570-1613

37. Paparoditis E and Politis D N (2000) The local bootstrap for kernel estimators under general dependence conditions. Annals of the Institute of Statistical Mathematics 52:139-159

38. Paparoditis E and Politis D N (2001a) The local bootstrap for Markov processes. Journal of Statistical Planning and Inference 108:301-328

39. Paparoditis E and Politis D N (2002) A Markovian local resampling scheme for nonparametric estimators in time series analysis. Econometric Theorey 17:540566

40. Paparoditis E and Politis D N (2003) Residual-based block bootstrap for unit root testing. Econometrica 71:813-855

41. Politis D N (2003) The impact of bootstrap methods on time series analysis. Statistical Science 18:219-230

42. Politis D N and Romano J P (1994) Large sample confidence regions based on subsamples under minimal assumptions. Annals of Statistics 22:2031-2050.

43. Politis D N and Romano J P (1994) The stationary bootstrap. Journal of the American Statistical Association 89:1303-1313

44. Politis D N, Romano J P and Wolf M (1997) Subsampling for heteroskestastic time series. Journal of Econometrics 81:281-317

45. Politis D N, Romano J P and Wolf M (1999) Subsampling. Springer Verlag, New York.

46. Politis D N, Romano J P and Wolf M (2004) Inference for Autocorrelations in the Possible Presence of a Unit Root. Journal of Time Series Analysis 25: 251-263.

47. Rajarshi M B (1990) Bootstrap in Markov-sequences based on estimates of transition densities. Annals of the Institute of Statistical Mathematics 42:253268

48. Robinson P (1983) Nonparametric estimators for time series. Journal of Time Series Analysis 4:185-207

49. Robinson P (2001) Testing for strong serial correlation and dynamic conditional heteroskedasticity in multiple regression. Journal of Econometrics 47:67-84

50. Romano J P and Wolf M (2001) Subsampling intervals in autoregressive models with linear time trend. Econometrica 69: 1283-1314 
51. Ruiz E and Pascual L (2002) Bootstrapping financial time series. Journal of Economic Surveys 16:271-300

52. Shepard N (1996) Statistical aspects of ARCH and stochastic volatility. In: Cox D R and Hinkley D V (eds) Time series models in Econometrics, Finance and other fields. Capman and Hall, London, p. 1-67

53. Shi S G (1991) Local bootstrap. Annals of the Institute of Statistical Mathematics 43:667-676

54. Straumann D (2005) Estimation in conditionally heteroscedastic time series models. Lecture Notes in Statistics, 181. Springer Verlag, Berlin

55. Taylor S (1986) Modelling Financial time series. Wiley, New York 
Index

paragraph, 9 
